\title{
Heisenberg's observability principle
}

\author{
J. Wolff \\ draft Nov 2013; for citations please refer to the published version
}

\begin{abstract}
Werner Heisenberg's 1925 paper "Quantum-theoretical re-interpretation of kinematic and mechanical relations" marks the beginning of quantum mechanics. Heisenberg famously claims that the paper is based on the idea that the new quantum mechanics should be "founded exclusively upon relationships between quantities which in principle are observable". My paper is an attempt to understand this observability principle, and to see whether its employment is philosophically defensible. Against interpretations of 'observability' along empiricist or positivist lines I argue that such readings are philosophically unsatisfying. Moreover, a careful comparison of Heisenberg's reinterpretation of classical kinematics with Einstein's argument against absolute simultaneity reveals that the positivist reading does not fit with Heisenberg's strategy in the paper. Instead the complain that electron orbits are unobservable should be understood as a criticism of the causal inefficacy of orbital electron motion in Bohr's atomic model. I conclude that the tacit philosophical principle behind Heisenberg's argument is not a positivistic connection between observability and meaning, but the idea that a theory should not contain causally idle wheels.
\end{abstract}

Keywords: quantum mechanics; Heisenberg; observability; positivism; Einstein. 


\section{Introduction}

Quantum mechanics was invented in the short period between 1925 and 1927. A pivotal contribution was made by the young Werner Heisenberg, in his celebrated 1925 paper "Über quantentheoretische Umdeutung kinematischer und mechanischer Beziehungen" [Heisenberg, 1925], which is usually taken to be the starting point of matrix mechanics. Famously, Heisenberg begins this paper proposing that the new quantum mechanics must be based only on relations between quantities that are in principle observable.

In the eyes of some of his colleagues at Göttingen, it was invoking this principle of observability, which allowed Heisenberg to make a crucial breakthrough, and which led to the development of quantum mechanics [Born, 1955]. By contrast, some of Heisenberg's contemporaries, notably Einstein, rejected the principle as misguided. ${ }^{1}$ These different assessments raise the question what role the principle of observability played for Heisenberg's argument, and what Heisenberg meant by 'observable' and 'unobservable' in the first place.

Two natural readings are to understand 'observable' either as what can be perceived or as what can be measured. On the first reading one might then attempt to defend the idea that only observable quantities should be admitted into physical theories on general empiricist grounds: we have better epistemic access to what can be perceived, and hence we should avoid including anything which cannot be perceived into our theories. On the second reading one might be tempted to connect measurability to meaning in what might be called a positivistic $^{2}$ manner: terms referring to quantities which are not measurable are (physically) meaningless, and hence should not be employed in theory construction.

The positivistic reading of Heisenberg's principle has seemed especially plausible in light of the then widespread positivist reading of Einstein's argument against absolute simultaneity in 1905, and the apparent commitment to positivist ideas of several of Heisenberg's close collaborators, as we will see in more detail in section three. Justifying the appeal to observables on either empiricist or positivistic grounds yields no philosophically satisfactory argument for Heisenberg's purposes, as will become clear in section four. It would seem then, that Heisenberg's paper does not succeed because of its appeal to observability, but in spite of it. Accordingly many recent interpretations try to save Heisenberg from embarrassment and view the appeal to observability as an ad hoc addition to the paper rather than as a guiding principle (e.g. Beller [1999] and Camilleri [2009]).

While I concede that observability may have played a less central role in Heisenberg's development of the mathematical techniques of the paper, it seems that declaring the principle to be ad hoc fails to explain the prominent role given to it in Heisenberg's presentation, and more importantly, it leaves Heisen-

\footnotetext{
${ }^{1}$ As Heisenberg vividly recalls in his autobiography 1969.

2'Positivistic' because of the emphasis the logical positivists put on the connection between empirical verifiability and meaning, not because all of the physicists who suggested principles along these lines were in fact positivists.
} 
berg's paper somewhat unmotivated. Heisenberg appears as the lucky kid who stumbled upon the key to solving the quantum puzzle, but why his approach succeeded remains unclear. In order to give an account of Heisenberg's success, we need to understand the role the observability principle was supposed to play in Heisenberg's argument. I argue that observability understood positivistically fails to do justice to the task, but that a principle like observability is nonetheless needed for Heisenberg's argument.

Through a careful reconsideration of the comparison of Einstein's and Heisenberg's way of reinterpreting classical kinematics I show in section five that unlike Einstein, Heisenberg makes no attempt to give a new empirical definition of his reinterpreted kinematic concepts. Instead his reinterpretation is purely mathematical. This suggests that the positivist reading of Heisenberg's project is not only unsatisfactory philosophically, but also unilluminating with regard to Heisenberg's actual strategy in the paper, which opens up the possibility of separating Heisenberg's appeal to observability from the positivistic interpretation of the principle.

In the final section of the paper I offer an alternative reconstruction of Heisenberg's argument as an objection to the introduction of causally idle wheels in Bohr's atomic theory. I show that while rejecting electron orbits and their properties on the basis of unobservability alone remains unsatisfactory, rejecting them as causally idle wheels is plausible. This allows us to acknowledge the importance of a principle playing the role the observability principle is supposed to play, without being forced to attribute to Heisenberg a dodgy positivistic argument.

\section{Heisenberg's 1925 paper}

Heisenberg's Umdeutung paper is justly regarded as the beginning of quantum mechanics proper. In his paper Heisenberg formulates the basis for what would soon become known as 'matrix mechanics' by replacing classical kinematic quantities like motion, understood as position as a function of time, by quantum theoretical representatives. Based on this replacement he uses classical equations of motion to describe the behavior of a simple model system: the one-dimensional anharmonic oscillator. In the course of his replacement of classical kinematic quantities, Heisenberg arrives at what would soon become a central principle of quantum mechanics: the non-commutativity of factors in the multiplication of certain quantum mechanical quantities. This multiplication rule, which Heisenberg initially regarded as a difficulty, led Born to interpret Heisenberg's calculations in terms of matrices. ${ }^{3}$

In the abstract to his paper, Heisenberg puts forward, as the organizing principle of his approach, what I will call Heisenberg's observability principle: "The present paper seeks to establish a basis for theoretical quantum mechanics founded exclusively upon relationships between quantities which in principle are

\footnotetext{
${ }^{3}$ See Mehra and Rechenberg [1982b] for a detailed discussion of the different aspects of the paper and its context.
} 
observable" [Heisenberg, 1925, 879]. ${ }^{4}$ In this section I will briefly show where Heisenberg appeals to this principle in his argument, and why this appeal has often seemed problematic. ${ }^{5}$

The first and by far most prominent appeal to observability occurs in the lengthy introduction of Heisenberg's paper, where he seems to use the principle to criticize the old quantum theory, i.e., the Bohr model of atomic structure. "It is well known that the formal rules which are used in quantum theory for calculating observable quantities such as the energy of the hydrogen atom may be seriously criticized on the grounds that they contain, as basic element, relationships between quantities that are apparently unobservable in principle, e.g., position and period of the revolution of the electron" [Heisenberg, 1925, 879]. Here we get a first indication of the quantities Heisenberg rejects as unobservable, namely quantities associated with electron orbits in Bohr's model, and of those he counts as observable, namely the energy of the hydrogen atom.

The reason Heisenberg offers for doubting that the former quantities will become observable later on is that the old quantum theory is beset with empirical problems and limited in scope. ${ }^{6}$ In light of these difficulties he suggests that the empirical successes of the old quantum theory ${ }^{7}$ are merely "fortuitous" and proposes "to try to establish a theoretical quantum mechanics, analogous to classical mechanics, but in which only relations between observable quantities occur" [Heisenberg, 1925, 880]. Heisenberg hence seems to appeal to observability both to criticize the old quantum theory, and to motivate his own novel approach.

That there were severe problems with the old quantum theory was widely acknowledged by 1925, so Heisenberg's extended criticism in the introduction is somewhat puzzling, unless we assume he is trying to establish the importance of the observability principle. The trouble is that this principle does not seem to contribute much to the overall argument, since the criticism of the old quantum theory rests ultimately on the empirical problems of the theory, not on the unobservability of the quantities in question. In fact, if anything the argument seems to go the other way around: the reason we should give up hope that the quantities will eventually become observable is precisely that the theory in which they occur is unpromising. ${ }^{8}$

Even if the appeal to observability does not seem essential to the criticism of the old quantum theory, it might still be used to motivate Heisenberg's replace-

\footnotetext{
${ }^{4}$ I am largely following the translation [Heisenberg, 1967] in van der Waerden 1967, except where indicated otherwise.

${ }^{5}$ I will only be concerned with Heisenberg's paper insofar as it is relevant to the understanding of the observability principle. For an excellent presentation of the model-based reasoning process Heisenberg may have gone through, see [MacKinnon, 1977]; for a technical reconstruction of Heisenberg's calculation see [Aitchison et al., 2004].

${ }^{6}$ Heisenberg lists in particular the problem of crossed fields and the inability to extend the quantum rules to atoms with more than one electron.

${ }^{7}$ What he presumably has in mind is the successful analysis of the hydrogen atom.

8 "This hope [to observe the quantities later] might be regarded as justified if the abovementioned rules were internally consistent and applicable to a clearly defined range of quantum mechanical problems" [Heisenberg, 1925, 880].
} 
ment of kinematic quantities like motion, $x(t)$. In quantum theory we cannot "associate the electron with a point in space, considered as a function of time, by the means of observable quantities" [Heisenberg, 1925, 881]; what we can do instead, however, is attribute emitted radiation to it. Heisenberg justifies the crucial step of using quantities associated with emitted radiation instead of classical kinematic quantities by appeal to the principle of using only observable quantities. The need to find a quantum theoretical representative for $x(t)$ arises only when we accept that being unable to assign position as a function of time on the basis of observable quantities constitutes a problem, and the only reason we seem to have for thinking that this is a problem is the principle of observability. Since this replacement is a crucial step in the paper, the principle of observability would seem to have a central role in Heisenberg's argument.

Despite this apparently crucial function of observability in justifying Heisenberg's own proposal, it has often been suggested that observability is not the motivation for Heisenberg's argument. Instead it seems that for much of the positive part of the paper it is the correspondence principle ${ }^{9}$ which drives the argument, even though Heisenberg seems reluctant to acknowledge its use. ${ }^{10}$

It is indeed plausible to suggest that Heisenberg was familiar with the particular techniques he employed in his reinterpretation from work he had done with Kramers in Copenhagen, ${ }^{11}$ and accordingly that those techniques were not exclusively motivated by the observability principle, but instead made heavy use of the correspondence principle. To pitch the observability principle against the correspondence principle, however, looks like a false dilemma. Even if Heisenberg's overall approach was shaped by the correspondence principle, this does not mean that the principle of observability did not have a key justificatory role to play in the argument. Without denying the importance of those other motivations and influences for Heisenberg's actual reasoning process, it seems to me that Heisenberg did feel a genuine unease about the use of electron orbits and their properties in the old quantum theory, and that he expressed this discomfort by describing these orbits as 'unobservable'.

Hints for this can be found in Heisenberg's letter to Wolfgang Pauli from July 9th 1925, which enclosed the Umdeutung manuscript: "all my own paltry efforts are aimed at killing completely the concept of orbits, which are not observable anyway, and to replace it suitably". ${ }^{12}$ The role of the observability principle, for Heisenberg, is to justify the elimination of the concept of electron orbits

\footnotetext{
${ }^{9}$ The exact interpretation of Bohr's correspondence principle is subject to much debate; for a recent survey of options see Bokulich [2010]. The use of the correspondence principle in the physics community seems to have been to start from relationships between classical quantities and to use them 'to guess' which 'corresponding' quantum relationships might hold among them. For a discussion of how Heisenberg's thinking about the correspondence principle evolved, see [Mehra and Rechenberg, 1982b].

${ }^{10}$ Beller [1999] speculates that Heisenberg may have been reluctant to emphasize his reliance on the correspondence principle because of its extensive use in the Bohr-Kramers-Slater theory, which had just been experimentally refuted.

${ }^{11}$ See Mehra and Rechenberg [1982b] for discussion.

${ }^{12}$ The German reads: "meine ganzen kümmerlichen Bemühungen gehen dahin, den Begriff der Bahnen, die man doch nicht beobachten kann, restlos umzubringen und geeignet zu ersetzen" [Pauli, 1979, 231; my translation].
} 
altogether, because Heisenberg suspects that electron orbits do not exist. The correspondence principle, as we shall see in more detail in section five, helped Heisenberg to find a suitable replacement, but that alone does not suffice to get rid of electron orbits. For that Heisenberg needs a reason to think that they have a feature that makes them unsuitable for physical theorizing, and he needs a philosophical principle to justify why having that feature makes them ineligible for physical theories. If this is the role of the observability principle, is observability the right principle to use? I will show first what observability might mean in the context of Heisenbergs paper, then I will show that the principle so understood does not work and does not reflect Heisenbergs strategy in the paper, and finally I propose a related, but different principle, which can play the required role, and which picks out the feature Heisenberg perhaps really thought made electron orbits suspect: they are causally idle wheels.

\section{Two readings of observability}

The idea that observability matters for science is not unique to Heisenberg. In this section we shall look at two interpretations of observability and observability principles, which can be found in discussions of physics prior to Heisenberg. Since at least the second of these principles was employed by friends of Heisenberg, it seems like a prima facie plausible candidate for understanding Heisenberg's own principle.

A traditional empiricist ${ }^{13}$ understanding of observability is perceptibility: only what can be perceived by the unaided senses is observable, everything else is unobservable. This conception of observability has certain advantages with respect to Heisenberg's argument. The position and period of revolution of an electron inside the atom are indeed not perceptible, and, more importantly, it is plausible to suggest that they will remain imperceptible. What is perceptible and what is not depends on our sensory organs and not on technical advances, and unlike technology, our sense organs change very slowly at best. It makes sense, then, to say that quantities like the position of an electron in an atom 'unobservable in principle'.

If we understand unobservable to mean imperceptible, Heisenberg's principle means that only relations among perceptible quantities may be admitted. It is difficult to see how such a principle could be defended, even from an empiricist point of view. ${ }^{14}$ Indeed, the quantities Heisenberg himself offers up as observable, like energy, are themselves not perceptible. Even the line spectra of atoms are only partly perceptible, since they extend beyond the visible realm.

\footnotetext{
${ }^{13}$ Heisenberg might have been familiar with this understanding of observability from the work of Ernst Mach, although in Heisenberg's recollection Mach had no decisive impact on his thinking [Kuhn et al., 1967, Session 1, 3-4].

${ }^{14}$ The limited force of this empiricist distinction is underscored by the fact that Bas van Fraassen, the most prominent contemporary defender of a distinction roughly along the lines sketched above, does not try to draw any ontological conclusions from this distinction, nor does he suggest that science should never involve reference to unobservables; instead he offers only an epistemological recommendation for the rest of us [van Fraassen, 1980].
} 
So it seems unlikely that Heisenberg's argument is going to succeed if 'observable' is taken to mean perceptible, even if a full-blown empiricist defense of the distinction and the restriction to perceptibles could be given.

A more likely source for Heisenberg's principle is Pauli's criticism of Hermann Weyl's unified field theory of 1918 [Weyl, 1920]. Pauli's objection concerned Weyl's use of the idea of an electric field inside the electron. As Pauli pointed out, "[f]or a physicist this [the field strength] is only defined as a force on a test-body, and since there are no smaller test-bodies than the electron itself, the concept of the electric field strength in a mathematical point seems to be an empty, meaningless fiction. One would like to hold on to the idea that only quantities observable in principle should be introduced into physics." [Pauli, 1919].

Pauli's objection to Weyl depends on a different reading of 'observable' from the one discussed above, since the electric field strength even outside the electron is not observable in the sense of being accessible to sense perception. Instead Pauli's notion of 'observability' has to do with measurement: what is measurable is observable, whether or not it is something we can see. The electric field inside the electron is unobservable in principle, in the sense of being unmeasurable in principle, because the one measurement procedure we have is systematically inapplicable due to the lack of an appropriate test-body.

Pauli does not offer any explicit reasons why introducing such in principle unmeasurable quantities into physical theories is problematic, but such reasons are easy to give on his behalf: unmeasurable quantities threaten the testability of the theory. If a theory stipulates that a certain relationship holds between several quantities, or that changes in a particular quantity are responsible for certain phenomena, then it is desirable to have some measurement procedure, direct or indirect, of that quantity in order to be able to generate specific predictions and to test them. If there are reasons to believe, not only that such measurements are unavailable, but that they are impossible, then this counts against the theory in question. It is not my concern here to evaluate whether this is in fact true for the debate between Weyl and Pauli, but we shall look carefully at whether Heisenberg might have objected to the old quantum theory on these grounds in the next section.

There is, however, another aspect to Pauli's objection, which seems to make his rejection of unobservable quantities more radical. When he calls the electric field inside the electron an "empty, meaningless fiction", he seems to suggest that a term referring to a quantity which cannot be measured is therefore meaningless, thereby connecting the measurability of quantities and testability of theories on the one hand with meaningfulness of terms occurring in the theory on the other. This sort of connection was of course defended by logical positivists, and it is natural to assume that at least one source for both Pauli and the positivists was Einstein's work on special relativity [1905]. According to this positivistic reading (e.g. Bridgman [1927], Reichenbach [1949]), Einstein's key insight in the 1905 paper had been that absolute simultaneity lacks 'physical meaning', precisely because it was not possible to give a measurement procedure 
for it even in a thought experiment. ${ }^{15}$

Much later, in his nobel lecture in 1954, Max Born would draw a direct comparison between Einstein's argument and Heisenberg's principle: "The principle states that concepts and representations that do not correspond to physically observable facts are not to be used in theoretical description. Einstein used the same principle when, in setting up his theory of relativity, he eliminated the concepts of absolute velocity of a body and of absolute simultaneity of two events at different places. Heisenberg banished the picture of electron orbits with definite radii and periods of rotation because these quantities are not observable [...]" [Born, 1955, 258-9]. Born here draws a direct connection not only between Einstein's and Heisenberg's arguments, but between the use of observability principles understood along positivistic lines in those arguments. A natural reading of Heisenberg's observability principle, then, is to take 'observable' to mean measurable, and to take the requirement of measurability to stem from an alleged connection between meaning and measurability.

\section{Problems with the measurability reading}

There are good reasons why the positivist reading of Heisenberg's argument seems appropriate. As we've seen in the previous section, this viewpoint was used by Pauli in his objection against Weyl. Heisenberg closely exchanged views and opinions with Pauli during much of his career, and he had talked with Pauli about the problem of electron orbits. ${ }^{16}$ Moreover, shortly after the publication of Umdeutung, in the defense of matrix mechanics against Schrödinger's competing wave-mechanics, Heisenberg seems to develop more explicitly positivistic views, culminating in his 1927 paper on the uncertainty principle. ${ }^{17}$ Nonetheless I will argue that observability understood as measurability does not suffice for role the observability principle is supposed to play in Heisenberg's argument. Observability understood as measurability cannot justify the elimination of electron orbits from the theory.

There are two general problems with understanding observability as measurability. First, it is somewhat ambiguous whether it is required that an actual measurement procedure is carried out, or whether it is enough to describe a possible procedure for carrying out such a measurement, even if actually carrying out this procedure is not currently feasible.

If the former, what is observable at any given moment is restricted primarily by the technology available, which does not seem to be the kind of restriction on which to build a strong ontological argument. Technology is likely to extend our reach, and so any distinction drawn between what is measurable and what is not would seem to be more an indication of our historically indexed limitations, and less an indication of a deep ontological difference among the entities which can currently be measured and those which cannot. Recall, though, that, for the

\footnotetext{
${ }^{15}$ I will come back to Einstein's argument in more detail below.

${ }^{16}$ See Kuhn et al. [1967, Session 5 p. 20]

${ }^{17}$ See Camilleri [2009] for a more nuanced view of the role of operationalism in that paper.
} 
argument in the paper, Heisenberg needs a principle which would allow him to conclude that electron orbits do not exist. There is no reason to 'kill' a concept when there is still hope it might soon apply to something measurable.

If the latter, on the other hand, the distinction does seem conceptual rather than practical, and so perhaps more suited as the basis of a philosophical argument. It also means, however, that it is more difficult to show that a given quantity fails to be observable on this standard. In particular, the quantities rejected by Heisenberg - the position and period of revolution of the electron inside the atom-might well turn out to be observable in this case, since it does seem possible to come up with a procedure which would determine them, even though the procedure can currently not be carried out. As suggested by Burkhard Drude, ${ }^{18}$ one might construct a gamma ray microscope, which would allow for radiation with sufficiently small wavelength to locate the electron inside the atom. Moreover, while nobody could claim to have measured the orbit of an electron inside the atom, there was good experimental evidence that electrons moved particle-like on trajectories outside of atoms: the tracks in Wilson cloud chambers. Accordingly, if all that is needed for electron orbits to count as observable would be for us to imagine extending our current measurement procedures, electron orbits seem likely to be observable.

Whether we take observability to require that something can be measured right now, or whether we are satisfied with the description of a measurement procedure in principle, it is not going to be enough to eliminate position and period of revolution of the electron.

The second problem with understanding 'observable' as measurable was forcefully made by Einstein in a conversation with Heisenberg. There Einstein argued against Heisenberg's employment of observability, that what is observable depends on the theory, and is not something which can be antecedently decided, as a means of motivating and developing the theory. It is easy to read this just along the lines of a general 'holism', according to which all observation is theory-laden and we have to give up the observable/unobservable distinction altogether. But there is a more specific, and perhaps more plausible reading of Einstein's objection, on which a theory determines what is observable through indirect measurement. ${ }^{19}$

One of the things our theories do is to offer up putative laws connecting directly measurable quantities to quantities not directly accessible in the hope of using the former quantity to measure the latter. Our ability to measure quantities thereby improves not just as our technology improves, but also as our theory advances. Einstein objects that quantum theory is not yet in a condition which allows a judgment about which quantities will ultimately become accessible through measurement, and given the dependence of what is measurable on the development of our theories, observability principles understood as measurability principles can never be used to determine the structure of the theory antecedently. In light of the fact that Heisenberg and many of his con-

\footnotetext{
${ }^{18}$ See Heisenberg $[1969,112]$ as well as Kuhn et al. [1967, Session 7, 26].

${ }^{19}$ See also Camilleri [2009] for further defense of this reading.
} 
temporaries had taken Einstein's own argument against absolute simultaneity to proceed from an observability principle, Einstein's objection to the principle seems especially significant, a point to which I will return in the next section.

If we understand Heisenberg's notion of observability as measurability, Heisenberg's argument is fundamentally flawed. What is measurable depends in no small part on us, on our theories and (technological) practices, and accordingly it would seem highly anthropocentric to assume that what is outside our epistemic reach does not exist. One might nonetheless attempt to strengthen the principle positivistically by suggesting that what is outside our (empirical) epistemic reach is not well understood, and it is really this lack of proper understanding which casts suspicion on the concepts involved. Indeed such a move might seem natural as a reading of Heisenberg's principle in light of the influence Pauli and Born had on his thinking and in light of the fact that he called his paper a 'reinterpretation' of classical kinematics. As I will show in the next section, however, this positivistic reading of the reinterpretation project in Heisenberg's paper is implausible.

\section{$5 \quad$ Rethinking the analogy with Einstein}

The positivistic reading had seemed plausible in no small part because of the widespread positivistic reading of Einstein's rejection of absolute simultaneity. In Born's understanding, the comparison with Einstein concerned the principle both Einstein and Heisenberg were thought to have employed: the observability principle.

There are other respects in which Einstein's and Heisenberg's paper might be compared as well. Mehra and Rechenberg [1982b, 303-304] list a number of similarities between the two papers: both Einstein and Heisenberg achieve a breakthrough by reformulating kinematics, they both base their papers on a few principles, and they end up with a surprising mathematical relation (Einstein with the addition law for light velocities, Heisenberg with the multiplication rule). Finally, the new theories approximate the classical ones in the limit, and each theory involves a new fundamental constant ( $c$ and $h$ respectively).

In light of these numerous similarities, and the fact that Heisenberg seems to have regarded Einstein's way of doing physics as paradigmatic, ${ }^{20}$ we should not conclude that Heisenberg did not intend his paper in analogy with Einstein's. Instead of trying to compare the alleged or actual principles at work in the two papers directly, perhaps a better starting point is provided by the task undertaken in the two papers: a reinterpretation of kinematics. This seems especially appropriate given that it is the explicit topic of Heisenberg's paper, as the title suggest. What is being redefined or reinterpreted in Einstein's and Heisenberg's papers, and how does the redefinition proceed?

In Einstein's case the redefinition concerns motion insofar as motion takes place in time. Mechanics describes motion mathematically by giving position

\footnotetext{
20 "I was impressed by Einstein's way of doing things, but not by Mach's." [Kuhn et al., 1967, Session 1, 3-4].
} 
as a function of time, but such a mathematical description only has "physical meaning" ("physikalischen Sinn"), once we understand what is meant by "time" [Einstein, 1905, 892]. For Einstein, then, the problem of redefinition concerns the problem of giving physical meaning to a mathematical description, and the concept in need of physical meaning is the concept of time. It quickly emerges, of course, that the real problem concerns not time but simultaneity in different locations, resulting in Einstein's famous observation that the definition of time depends on the notion of a system at rest, from which, together with the light principle, it follows in turn that we cannot think of simultaneity as being defined absolutely.

In giving his definitions Einstein proceeds by providing "imaginary physical experiments", ${ }^{21}$ which reveal the assumptions involved in making claims about simultaneous events at distant locations. Giving physical meaning to a mathematical description involves the description of experiments, or better, measurement procedures, which seems to support a positivist reading of the argument. The positivist reading assumed that Einstein's key innovation was to demand this kind of physical meaning in the first place, and that conversely, a physical meaning along these lines had been absent from Newtonian mechanics. On this reading, absolute time and simultaneity lack physical meaning because no measurement procedure can be described for them, which makes them, in one sense, unobservable. Understood this way, Einstein's argument does involve a 'principle of observability': only observable quantities may be admitted into the theory.

This reading, however, rests on a misunderstanding. As Robert DiSalle [2008] has argued, the problem was not that simultaneity lacked an empirical definition along the lines given by Einstein, but that its empirical definition assumed that Newton's velocity addition law would hold for light also, and that light could accordingly be treated as a proxy measure for an instantaneous signal. Use of an instantaneous signal would of course permit the synchronization of clocks across spatial distances, and thereby allow for an empirical definition of absolute simultaneity. Electromagnetism reveals that this treatment of light is impermissible: light travels at a fixed velocity which cannot be increased by emitting it from a moving body, and therefore instantaneous signaling cannot be approximated using light. Einstein's empirical definition of simultaneity must therefore be understood as redefinition in response to the fact that empirical assumptions made by the previous definition turned out to be false.

Heisenberg's redefinition also concerns kinematical quantities, and it is selfconsciously a reinterpretation ("Umdeutung"). ${ }^{22}$ Like in Einstein's case the quantity to be redefined appears to be motion, or position as a function of time $x(t)$. But here the papers diverge. Heisenberg's worry does not concern time, but the possibility of assigning a position as a function of time to an electron. Such an assignment, Heisenberg claims, is not possible on the basis of observable

\footnotetext{
${ }^{21}$ I follow the translation in [Einstein, 1952]; the German is "gedachte physikalische Erfahrungen" [1905, 892].

${ }^{22}$ Darrigol [1992, 274] also emphasizes the importance of "Umdeutung" in the title, tracing it back to Arnold Sommerfeld's use of the term [1922].
} 
quantities, which makes position an ineligible quantity by the standards of the observability principle.

Appeal to observability might suggest an attempted positivistic move, perhaps based on the positivist reading of Einstein's argument above: $x(t)$ for electrons needs to be redefined, because in the absence of a link with observable quantities, the quantity $x(t)$ lacks physical meaning. If this was the intent, we should expect Heisenberg to follow up by offering physical thought experiments providing an empirical definition of electron motion in quantum mechanics. What follows, however, is nothing of the sort.

Instead of an empirical redefinition by means of thought experiments, Heisenberg offers a mathematical redefinition using the correspondence principle, specifically appealing to the correspondence between the quantities associated with the emitted radiation (i.e. frequencies and amplitudes) and the harmonics of periodic motion in classical mechanics. ${ }^{23}$ The quantum theoretical representative for the quantity $x(t)$, Heisenberg suggests, should be the ensemble of quantities $\mathfrak{A}(n, n-\alpha) e^{i \omega(n, n-\alpha) t} \cdot{ }^{24}$ This ensemble consists of transition amplitudes and phases, which unlike their classical counterparts depend on two 'stationary states' $n$ and $n-\alpha$. Because of this dependence on two states it is not possible to represent

$$
x(n, t)=\sum_{-\infty}^{+\infty} \alpha \mathfrak{A}_{\alpha}(n) e^{i \omega(n) \cdot \alpha t}
$$

as one would for periodic motion in the classical case.

Representing $x(t)$ as $\mathfrak{A}(n, n-\alpha) e^{i \omega(n, n-\alpha) t}$ instead, Heisenberg is faced with a new problem: the phase $e^{i \omega(n, n-\alpha) t}$ is complex, thereby raising a question about the "physical meaning" [Heisenberg, 1925, 882] both of the phase and the amplitude containing it. Heisenberg solves this problem by devising a rule to arrive at the quantum representative for the squared quantity $[x(t)]^{2}$, which will be real instead of complex. Since the individual (complex) phases still contribute to the resulting quantity, Heisenberg concludes that the phases have physical significance even in quantum theory. ${ }^{25}$ However, as Heisenberg remarks, "a geometrical interpretation of such quantum-theoretical phase relations in analogy with classical theory initially seems hardly possible" [Heisenberg, 1925, 883].

If we follow Heisenberg's Umdeutung of kinematics, motion in quantum mechanics cannot be understood as a change of position over time, but at least in

\footnotetext{
${ }^{23}$ Darrigol [1992, 275] points out, rightly, that the correspondence principle played hence a central role in Heisenberg's derivation, but follows others in concluding that this therefore limits the role the observability principle played in the paper. But there seems to be no particular reason to think that the correspondence principle and the observability principle are in competition.

${ }^{24}$ In Heisenberg's own recollection of how he arrived at this representation of $x(t)$ he freely admits that the details of how this representation should pan out or why it should work were quite vague: "Well, it $[x(t)]$ means certainly some kind of radiation, so all these frequencies which are possibly emitted do somehow represent this $X(t)$. "Somehow represents" was, of course, a very vague term but it wasn't more than that." [Kuhn et al., 1967, Session 7, 9]

${ }^{25} \mathrm{It}$ is of course in moving from the representative of $x(t)$ to that of $[x(t)]^{2}$ that Heisenberg discovers the famous multiplication rule.
} 
this paper, ${ }^{26}$ Heisenberg seems to see no need to give physical meaning or an empirical interpretation to this new mathematical characterization of motion beyond constructing it in analogy with classical mechanics. The only challenge to the physical significance of his new quantum theoretical quantities Heisenberg seems to be willing to countenance is that the individual phases are complex. As soon as that threat is (mathematically) removed, Heisenberg moves on, despite the fact that "a geometrical interpretation" of the newly devised phase relations is not possible. If Heisenberg had thought that the problem with position and period of revolution of the electron in Bohr's model concerned the physical meaning of these terms, he should have been much more worried about the fact that his own reinterpretation of motion lacks a geometrical interpretation. It seems natural to conclude from this that empirical or physical meaning was not the concern of Heisenberg's Umdeutung.

What persuaded him and others that these representatives made sense physically was that they allowed the formulation of equations of motion for simple systems (in Heisenberg's paper the one-dimensional anharmonic oscillator) by replacing the classical kinematic quantities with the quantum theoretical representatives developed in the earlier parts of the paper. At least for simple systems Heisenberg could show that energy would be conserved under quantum conditions. Heisenberg had thereby succeeded in giving a mathematical description of the 'motion' of electrons in atoms. Recall that Einstein started from such a mathematical description, demanded an assignment of physical meaning, and proceeded to provide it by developing his famous thought experiments. From a positivistic perspective, then, Heisenberg's redefinition remains incomplete.

Heisenberg's 1927 paper on the Uncertainty Relations, by contrast, seems to aim to close the gap. While a geometrical interpretation of the mathematical descriptions provided by quantum mechanics was still out of reach, Heisenberg there offers an experimental illustration ${ }^{27}$ of the uncertainty relations, which are a consequence of the multiplication rule introduced in 1925 . In a sense, ${ }^{28}$ then, Heisenberg's 1927 paper completes the project begun in 1925.

The absence of thought experiments and operationalizations of the newly introduced quantum mechanical motion strongly suggests that reading the operationalism of the later paper into the earlier one would be a mistake. Heisenberg's concern in 1925 is to come up with a mathematical description of electron motion in atoms, not to give physical meaning to the fledgling quantum mechanics. Accordingly we should not feel bound by later operationalist tendencies in

\footnotetext{
${ }^{26} \mathrm{As}$ one reviewer helpfully points out, Heisenberg did worry about the question of how to interpret the newly found quantum mechanical expressions in his correspondence of the same year (e.g. Heisenberg to Pauli, 24th June 1925 [Pauli, 1979, 228]). It seems to me that this strengthens the idea that the Umdeutung paper is concerned with a different kind of reinterpretation, since Heisenberg himself does not seem to think it provides a sufficient response to the question of empirical meaning.

${ }^{27}$ This reading of the famous gamma ray thought experiment has been defended by Kristian Camilleri [2009].

${ }^{28}$ In the 1927 paper Heisenberg must be seen as pursuing multiple goals at once, which accounts for the often noted ambiguities and near-contradictions in that paper, see [Beller, 1999] for discussion.
} 
Heisenberg's writing to interpret observability in his 1925 paper along positivistic lines. Is there an alternative way of understanding Heisenberg's argument?

\section{Observability and causally idle wheels}

For clues on how to understand observability, let's focus on the role observability seems to have played in Heisenberg's paper. As I have already pointed out in section one, the role of observability was to justify the need for a reinterpretation of kinematics. Heisenberg seems to have seen a very close connection between what is observable and what is real, and this connection was plausibly the result of his exposure to the discussions in Göttingen:

When one spoke about special relativity, people always said, "Well, there was this very famous point of Einstein that one should only speak about those things which one can observe, that actually the time entering in the Lorentz transformation was the real time." And in some way that was an essential turn which Einstein had given to the Lorentz idea. Lorentz had the right formulas, but he thought that was the apparent time. Einstein said, however, "There is no apparent and no real time; there is just one real time, and that is what you call the apparent time." So this turning of the picture by saying the real things are those which you observe and everything else is nothing was in the minds of the Göttingen people. [Kuhn et al., 1967, Session 5, 18]

This connection between what is real and what is observable can easily be read along positivistic lines. Yet, as we have seen above, Heisenberg's redefinition procedure is radically different from Einstein's, so even if he mistakenly thought that unobservability permitted the empirical redefinition of terms, this would still not explain his procedure in 1925. For Heisenberg, the unobservability of position and period of the electron inside the atom justifies the mathematical reinterpretation of the motion of electrons. What Heisenberg needs is a reason to 'kill completely' the concept of an electron orbit, and for that he needs a reason to think that the quantities associated with electron orbits do not exist. Can such a reason be given on Heisenberg's behalf, even if he himself failed to do so? I suggest that Heisenberg's dissatisfaction with electron orbits and their associated quantities is justified ultimately by the causal inefficacy of those quantities in Bohr's atomic theory.

Since in Bohr's model the rotational frequencies of electron orbits did not correspond to the frequencies of the emitted light, the source of the radiation and its properties (wavelength, intensity, polarization and phases) remained obscure. Bohr's division between classical mechanics, which was supposed to describe the motion of the electron in the atom, and a quantized electrodynamics, meant that any hope for a mechanical explanation of the features of the emitted radiation must be give up. This is perhaps why Heisenberg links 
unobservability to "Unanschaulichkeit" in the introduction to his paper. ${ }^{29}$

On Bohr's model, not only was there no mechanism to explain the properties of the emitted light, since light was emitted (or absorbed) in non-classical quantum jumps, there was not even hope to restore anything akin to a mechanical explanation, because Bohr had severed the connection between classical mechanics and quantum theory in order to save the stability of electron orbits. The only solution Heisenberg saw to restore at least the hope for a mechanical explanation was to abandon the concept of electron orbits, but more specifically, to reject the connection between orbits and mechanics. The new mechanics had to be created using the properties of the emitted radiation, not the properties of the alleged orbits. If a mechanics could be "fabricated" (Heisenberg to Pauli June 211925 [Pauli, 1979, 221]) which used only features of the emitted radiation, a new very tight connection between electrodynamics and the novel 'quantum mechanics' would have been forged.

Just like Einstein put Lorentz's theory on its head by replacing 'real' time by 'apparent' time, Heisenberg put Bohr's theory on its head by replacing orbital frequencies by emitted frequencies, that is, by redefining kinematic concepts by means of electrodynamic ones, which had already been subjected to quantum considerations. This also provides a clue to why Heisenberg's treatment provided the foundations of quantum mechanics: he chose the part of Bohr's theory that had been given a quantum treatment as the basis of the mechanics to follow. What would become a key feature of quantum physics, namely the multiplication rule, results from the dependence of quantum mechanical quantities on two stationary states, a feature which could first be seen in the frequencies of the emitted light. Heisenberg was successful because he picked those quantities which were already 'quantized' as the basis for his new mechanics, not because those quantities were observable.

If my reconstruction is correct, the reason Heisenberg rejected electron orbits and their associated quantities was that Bohr's theory rendered them inevitably causally inefficacious. Such principled causal inefficacy is a problem for a theory, since it both leaves something causally unexplained, and it introduces causally idle wheels, which seem unconnected and unconnectable to any other parts of the theory through dynamical laws. Orbits in Bohr's theory were especially weird, since they were themselves supposed to be stationary (electron motion on those orbits notwithstanding) and yet they were needed in the explanation of line spectra by providing the starting and endpoints of electron 'jumps'. Orbits thereby had to be understood as physically significant for the explanation of line

\footnotetext{
29 "It is well known that the formal rules which are used in quantum theory for calculating observable quantities such as the energy of the hydrogen atom may be seriously criticized on the grounds that they contain, as basic element, relationships between quantities that are apparently unobservable in principle, e.g., position and period of the revolution of the electron. Thus these rules evidently lack an intuitive [anschauliche] physical foundation, unless one still holds out hope that these hitherto unobservable quantities might become experimentally accessible later on." [Heisenberg, 1925, 879; my translation and emphasis] Since on Bohr's model electron motion was geometrically represented (as an orbit), Heisenberg's complaint is puzzling, unless we take it to be a complaint about the inevitable absence of a fully mechanical model.
} 
spectra, yet the kinematic quantities associated with them had to be understood as causally inefficacious. By rejecting electron orbits, Heisenberg had put his finger right on the central paradox in Bohr's model.

One might worry that breaking one causal link, namely that between orbital electron motion and radiation, does not rule out the possibility of other causal links being discovered. So in what sense does the 'no causally idle wheels' principle fare better than the original observability principle?

Given the specific role of electron orbits in Bohr's model, Heisenberg would have had good reason to believe that no such as of yet unknown causal links will emerge. By insisting that the energy of each orbit must be stable, Bohr had built into his model assumptions which made it difficult to see how the motion of electrons on orbits should have any causal effects whatsoever. Energy transfer is arguably a necessary condition for physical causation, ${ }^{30}$ and as long as the electrons are traveling on Bohr's orbits, the energy is by stipulation stable. Since this is built into the very basis of Bohr's model, it makes sense to think that on Bohr's theory the kinematic quantities of electron orbits must be causally inefficacious.

Moreover, since any theory which does not assume orbits to be stationary leads to unstable atoms, Heisenberg would seem to be justified in thinking that electron orbits are causally inefficatious in principle: they cannot consistently be conceptualized in any other way. Perhaps this is how we should understand his letter to Pauli from June 21st 1925, where he writes: "If something like mechanics were to hold, it would be inconceivable how there could be atoms; instead what holds is a different [kind of mechanics], a 'quantum mechanics', and one should only be surprised that the hydrogen atom happens to agree with something classical with respect to the energy constant." 31

To think of the motion of the electron inside the atom as an observable quantity it had to be divorced from the geometrical representation of that motion, because it was the electrodynamic quantities which were associated with energy transfer, not the kinematical ones attributed to 'classical' electron motion. But if that is the real reason for rejecting electron orbits, why did Heisenberg not say so?

While it is true that Heisenberg does not make any reference to causal inefficacy in the paper itself, his letter to Pauli where he first articulates his basic principle can actually be read as making a claim about causal relevance. "The principle is this: Only relationships between quantities in principle controllable may occur in the calculations for any kind of quantities like energy, frequency and so forth." 32 It is very plausible to link this to the observability principle, which occurs in the paper, but it is interesting that Heisenberg here refers to the

\footnotetext{
${ }^{30}$ See Dowe [2000] for a recent philosophical treatment of that idea.

31 "Wenn so etwas, wie die Mechanik, gälte, würd man nie verstehen können, daß es Atome gibt; es gilt eben eine andere, eine 'Quantenmechanik', und man muß sich nur drüber wundern, daß das Wasserstoffatom zufällig hinsichtlich der Energiekonstante mit etwas klassischem übereinstimmt" [Pauli, 1979, 219], my translation.

${ }^{32}$ The German reads: "Grundsatz ist: Bei der Berechnung von irgendwelchen Größen, als Energie, Frequenz u.s.w. dürfen nur Beziehungen zwischen prinzipiell kontrollierbaren Größen vorkommen" [Pauli, 1979, 227; my translation].
} 
ability to control such quantities. For experimental control has recently [Woodward, 2003] been linked to an understanding of causation frequently found in the sciences. Perhaps Heisenberg's unease about electron orbits results not from their unobservability, but their causal inefficacy and the resulting impossibility to design experiments in which it is possible to manipulate them.

Part of the historical reason why Heisenberg ultimately chose observability over controllability as his guiding principle might be that Heisenberg was in fact too deeply steeped in the discussions at Göttingen, which focused on observability. Since there is a connection between causal inefficacy and observability, this might not have seemed to matter. A quantity which is causally inefficacious in principle is thereby also unobservable in principle, since in order to be observable, whether in the sense of being measurable or in the sense of being perceptible, a quantity has to be causally active. Perception and measurement are themselves causal processes, in which causally idle wheels by assumption cannot participate. In this sense the quantities associated with electron orbits on Bohr's atomic model were indeed unobservable.

Being a causally idle wheel entails being unobservable, but not vice versa, however. If Heisenberg arrived at his conclusion that electron orbits are suspicious and possibly unreal on the basis of their causal inefficacy, then it seems he should have employed a stronger, and overall more plausible principle for concluding that electron orbits are unreal than the observability principle. In that case his connection with the more positivistically inclined Göttingen school of thought inadvertently weakened his argument. Heisenberg had identified the suspect feature - being causally idle - but used the wrong principle to defend it. Saying that something is unobservable or unmeasurable always makes reference to our sensory, technical, and theoretical abilities. Saying that something is causally inefficacious, however, has no such anthropomorphic restriction.

Does that mean the observability principle was ad hoc after all? To claim that the observability principle is ad hoc suggests to me that the principle can be dispensed with without any loss to the argumentative structure of the paper. That, I believe, is not correct, since Heisenberg needs an argument to support the claim that there is something fundamentally wrong with the kinematics of atoms as understood by Bohr, and that role cannot be played by the correspondence principle alone. There is nonetheless something to that particular complaint about observability: Heisenberg's dissatisfaction with electron orbits probably was not due to their unobservability alone, and the observability principle does not suffice to make Heisenberg's case in the paper. Because of the connection between causal inefficacy and unobservability, however, we might understand Heisenberg's appeal to observability as his, ultimately inadequate, attempt to articulate his deeper suspicion against electron orbits.

\section{Conclusion}

I have argued that Heisenberg's observability principle should not be understood along positivistic or empiricist lines. Such an interpretation does not yield a 
satisfactory argument for Heisenberg's purposes in his 1925 paper. Instead, looking more closely at the alleged analogy with Einstein's argument against absolute simultaneity, I suggested that Heisenberg's observability principle is a way of framing his dissatisfaction with a fundamental aspect of Bohr's atomic model: the causal inefficacy of the electron orbits. Since causal inefficacy of a quantity entails its unobservability, Heisenberg's claim that position and period of revolution in the atom are unobservable in principle is justified.

This should not lead us to think that Heisenberg was committed to the idea that the terms 'electron orbit' and 'period of revolution' were meaningless. To say so seems implausible both because we do seem to have an understanding of them in terms of classical kinematic concepts and because we can in fact imagine measurement procedures for them. Fortunately the connection with meaning (or meaninglessness) is not needed for the purposes of Heisenberg's argument. Causally idle wheels are a disadvantage in a physical theory, even if we understand the terms which purport to refer to them. Moreover, the details of Heisenberg's reinterpretation show, that he is not concerned with physical meaning in the sense demanded by Pauli and Einstein. Accordingly we can both understand and defend Heisenberg's argument without reference to positivistic theories of meaning.

\section{References}

Ian J.R. Aitchison, David A. MacManus, and Thomas M. Snyder. Understanding Heisenberg's "magical" paper of July 1925: A new look at the calculational details. American Journal of Physics, 72:1370-1379, 2004.

Mara Beller. Quantum Dialogue - the making of a revolution. The University of Chicago Press, 1999.

Alisa Bokulich. Bohr's correspondence principle. In Edward N. Zalta, editor, The Stanford Encyclopedia of Philosophy. Winter 2010 edition, 2010.

Max Born. The statistical interpretation of quantum mechanics. In Nobel Foundation, editor, Nobel Lectures: Physics 1942-64, Nobel Lecture. Elsevier Publishing Company, 1955.

Bridgman. The Logic of Modern Physics. MacMillan, 1927.

Kristian Camilleri. Heisenberg and the interpretation of quantum mechanics. Cambridge University Press, 2009.

Olivier Darrigol. From c-numbers to q-numbers: classical analogy in the history of quantum theory. University of California Press, Berkeley, 1992.

Robert DiSalle. Understanding Space-Time: The Philosophical Development of Physics from Newton to Einstein. Cambridge University Press, 2008. 
Phil Dowe. Physical Causation. Cambridge University Press, 2000.

Albert Einstein. Zur Elektrodynamik bewegter Körper. Annalen der Physik, pages 891-921, 1905 .

Albert Einstein. On the electrodynamics of moving bodies. In Lorentz H.A., A. Einstein, H. Minkowski, H. Weyl, W. Perrett, and Jeffery G.B., editors, The Principle of Relativity. Dover, 1952.

Werner Heisenberg. Über quantentheoretische Umdeutung kinematischer und mechanischer Beziehungen. Zeitschrift für Physik, XXXIII, June 1925.

Werner Heisenberg. Quantum-theoretical re-interpretation of kinematic and mechanical relations. In B. L. van der Waerden, editor, Sources of Quantum Mechanics, chapter 12. North-Holland Publishing Company, 1967.

Werner Heisenberg. Der Teil und das Ganze. In Walter Blum, Hans-Peter Dürr, and Helmut Rechenberg, editors, Gesammelte Werke - Abteilung C: Allgemeinverständliche Schriften, volume 3. Piper, 1969.

Thomas S. Kuhn, John L. Heilbron, Paul Forman, and Lini Allen. Sources for History of Quantum Physics. Archive for the History of Quantum Physics at the American Philosophical Society, Philadelphia, memoir 68 edition, 1967.

Edward MacKinnon. Heisenberg, Models, and the rise of Matrix Mechanics. Historical Studies in the Physical Sciences, 8:137-188, 1977.

Jagdish Mehra and Helmut Rechenberg. The Historical Development of Quantum Theory, Vol.2. Springer, 1982b.

Wolfgang Pauli. Merkurperihelbewegung und Strahlenablenkung in Weyls Gravitationstheorie. Verhandlungen der Deutschen Physikalischen Gesellschaft, 21:742-750, 1919.

Wolfgang Pauli. Wissenschaftlicher Briefwechsel mit Bohr, Einstein, Heisenberg u.a., volume I - 1919-1929. Springer, 1979.

Hans Reichenbach. The philosophical significance of relativity. In P.A. Schilpp, editor, Albert Einstein: Philosopher-Scientist, pages 289-311. Open Court, 1949.

Arnold Sommerfeld. Quantentheoretische Umdeutung der Voigt'schen Theorie des anomalen Zeemaneffektes vom D-Linientypus. Zeitschrift für Physik, 8: 257-272, 1922.

B. L. van der Waerden, editor. Sources of Quantum Mechanics. North-Holland Publishing Company, 1967.

Bas van Fraassen. The Scientific Image. Clarendon Press, Oxford, 1980. 
Hermann Weyl. Gravitation und Elektrizität. In Lorentz, Einstein, Minkowski, and Weyl, editors, Das Relativitätsprinzip. Teubner, Leipzig, 1920.

James Woodward. Making Things Happen. Oxford University Press, 2003. 